\title{
ANÁLISIS DEL PERFIL Y SATISFACCIÓN DEL TURISTA QUE VISITA MONTAÑITA-ECUADOR
}

\author{
Wilmer Carvache Franco* \\ Mónica Torres-Naranjo** \\ Mauricio Carvache Franco ${ }^{* * *}$ \\ Escuela Superior Politécnica del Litoral, ESPOL. Guayaquil-Ecuador
}

\section{RESUMEN}

La comuna de Montañita se encuentra ubicada en la parroquia de Manglaralto, provincia de Santa Elena en Ecuador, es un destino turístico de sol y playa, donde la práctica del surf es considerada uno de sus principales atractivos. Este trabajo se propone analizar el perfil de los turistas extranjeros que visitan Montañita, así como las motivaciones que los llevan a visitar el destino y la percepción de satisfacción de los servicios turísticos ofertados en este balneario. La recolección de la información se realizó "in situ". Los resultados sugieren evidencia importante acerca del perfil del turista que visita Montañita, hombres y mujeres llegan al destino de forma variada procedentes de varios continentes, en su gran mayoría son solteros, las motivaciones más valoradas fueron disfrutar del sol y la playa, seguida de descansar, lo que evidencia que, la playa, se convierte en el mayor atractivo turístico de este lugar. Las variables más valoradas en la satisfacción de los servicios hoteleros fue la ubicación y en los servicios de restaurantes fue la calidad de las comidas y bebidas. Los resultados obtenidos servirán para que los prestadores de servicios turísticos diseñen productos y ofrezcan servicios acorde a la demanda.

Palabras claves: Perfil del turista, motivación, satisfacción, turismo Ecuador, Montañita.

Recibido: 28 de noviembre de 2015

Devuelto para su revisión: 2 de mayo de 2016

Aceptado: 9 de julio de 2016

* Escuela Superior Politécnica del Litoral, ESPOL, Facultad de Ingeniería Marítima, Ciencias Biológicas Oceánicas y Recursos Naturales. Campus Gustavo Galindo, km 30.5. Vía Perimetral, P.O. Box 09-01-5863. GUAYAQUIL (Ecuador). E- mail: wcarvach@espol.edu.ec

** Escuela Superior Politécnica del Litoral, ESPOL, Facultad de Ciencias Sociales y Humanísticas. Campus Gustavo Galindo, km 30.5 Vía Perimetral, P.O. Box 09-01-5863.GUAYAQUIL (Ecuador). E- mail: mmtorres@ espol.edu.ec

*** Escuela Superior Politécnica del Litoral, ESPOL, Oficina de admisiones. Campus Gustavo Galindo, km 30.5 Vía Perimetral, P.O. Box 09-01-5863. GUAYAQUIL (Ecuador). E- mail: scarvach@espol.edu.ec 


\title{
Profile and satisfaction analysis of tourists who visit Montañita-Ecuador
}

\begin{abstract}
Montañita commune is located in the parish of Manglaralto, Santa Elena province in Ecuador, is a tourist destination of sun and beach, where surfing is considered one of its main attractions. This paper analyzes the profile of foreign tourists visiting Montañita, as well as the motivations that led them to visit the destination and the perception of satisfaction of tourist services offered in this beach town. The data collection was carried out "in situ". The results suggest important evidence about the profile of tourists visiting Montañita, men and women arrive at the destination in different ways from several continents, mostly are singles, whose most valued motivations were enjoying the sun and the beach, followed by resting, which shows that, the beach becomes the biggest tourist attraction of this place. The variables most valued about hotel services satisfaction was the location, and about restaurant services was the quality of food and drinks.The results will assist the tourism service providers to design products and offer services according to demand.
\end{abstract}

Keywords: Tourist profile, Motivation, Satisfaction, Ecuador tourism, Montañita.

\section{INTRODUCCIÓN}

El turismo de sol y playa constituye una tipología que mueve muchos viajeros hacia un destino que mantiene condiciones naturales y que presenta facilidades para los turistas. Debido a que cada vez más personas están viajando al extranjero de vacaciones, con un crecimiento del $34 \%$ entre el año 2007 y 2014, en cuanto a las vacaciones de sol y playa se presenta un crecimiento del $39 \%$ en el mismo periodo, manteniendo la cuota de mercado más alta con el $29 \%$ en comparación a todos los tipos de viajes de vacaciones (ITB World Travel Trends Report 2015/2016). En este sentido, los destinos turísticos buscan cada día ser más competitivos en el mercado y, por ello, un destino con un potencial turístico para la práctica de deportes como el surf es muy importante dentro de la oferta turística de cualquier país, como sería el caso de Ecuador. En este contexto Ayala, Martín y Masiques (2003), manifiestan que se debe diversificar la oferta de productos turísticos e incrementar los parámetros de calidad tomando en cuenta factores medioambientales. Por lo tanto en destinos de sol y playa, como el destino objeto de esta investigación, es necesario trabajar en beneficio de la calidad, para lo cual es clave desarrollar estrategias en el mejoramiento continuo de los productos turísticos.

En ese sentido la Comuna de Montañita, como un tradicional destino de sol y playa en Latinoamérica, está presente como opción para los turistas que desean visitar la costa Ecuatoriana. El reconocimiento que tiene Montañita es cada vez mayor debido a las diferentes actividades que se pueden realizar en este destino turístico, entre otras la práctica del surf. Así, Montañita es considerada como la capital del surf de Ecuador, la playa es amplia y con un fuerte oleaje el cual es favorable para las prácticas y competencias del surf. Siguiendo a Espejo (2011) se coincide que estas condiciones geográficas y naturales se deben aprovechar para impulsar la economía de este destino durante todo el año. 
Por ello, el objetivo de este artículo es presentar las principales características del perfil del turista extranjero que visita Montañita, además se identificarán las principales motivaciones de los viajeros, y la satisfacción de los servicios turísticos que se ofrecen en este destino. A continuación de la introducción, en el segundo apartado se realiza la revisión de la literatura; para continuar en el tercer apartado con la descripción del área geográfica del objeto de estudio. En el cuarto segmento se expone la metodología y en el quinto apartado se describen los resultados de la investigación y su discusión. Finalmente el artículo muestra las conclusiones de la investigación y las referencias bibliográficas utilizadas.

\section{REVISIÓN DE LITERATURA}

En diferentes estudios se ha analizado el perfil y la demanda de los turistas (entre otros, González, Sánchez y Sanz, 2006; Brida, Aguirre y Velásquez, 2010; De la Torre, 2011; Arizón, Garcés y Sangrá, 2012; López-Guzmán, Ribeiro, Orgaz-Agüera y Marmolejo Martín, 2015; Pérez y Lois, 2016), identificando el perfil socio-demográfico de los viajeros, las motivaciones y las valoraciones que realizan respecto a determinadas variables relacionadas con el turismo, lo que revela la importancia de este tipo de investigaciones.

En ese sentido, el estudio de las motivaciones permite entender mejor las expectativas, las necesidades y los objetivos de los turistas y es un elemento fundamental para diseñar estrategias de marketing y ayudar al posicionamiento y competitividad de los agentes implicados en la actividad turística (Beltrán Bueno, 2014). No obstante, otras investigaciones realizadas sobre la motivación turística (entre otros, Urry, 1994; Cohen, 2005) plantean la influencia de una amplia variedad de factores subjetivos en la configuración del comportamiento turístico, que al interrelacionarse con factores objetivos (demográficos y socioeconómicos), modulan la evaluación y las preferencias por las distintas alternativas de consumo turístico. Así, el análisis de las motivaciones se constituye como uno de los elementos más importantes para comprender las razones que llevan a una persona a demandar un determinado producto o servicio. Siguiendo a Martínez (2011), para realizar un análisis del consumidor en cuanto a su comportamiento, es necesario diferenciar los factores externos de los internos, los factores internos son aquellos que dependen del consumidor, como la personalidad, actitudes, creencias, y entre los factores externos se destacan la cultura y estilos de vida, lo que parecería indicar que en la visita a un destino y su retorno depende no únicamente de sus atractivos como la playa o de sus servicios y su infraestructura, sino que existe una influencia de otros factores.

En destinos como Montañita, es razonable pensar que las características propias del lugar se configuran como un factor determinante entre aquellos turistas cuyas motivaciones sean de Sol y Playa. En ese contexto, la principal motivación del turista de sol y playa es el descanso y el esparcimiento, dado a que se incluye dentro del turismo de ocio (Martínez, 2001; Rivas, 2004; Bigné, Sánchez y Currás, 2007). Según González et al (2006) las principales motivaciones de los turista de sol y playa es el descanso y el relax seguidos por la diversión y el entretenimiento, por lo que es importante establecer cuáles son las principales motivaciones de un destino de sol y playa para poder mejorar su oferta turística. 
Asimismo, uno de los tópicos más abordados en el campo de las investigaciones en turismo es la satisfacción (Kozak, Bigné y Andreu, 2003). De acuerdo a la definición de Oliver (1980), esta se puede definir como la evaluación global que hace el cliente del servicio recibido en comparación con el servicio esperado. Es fundamental establecer si el turista regresaría al destino y si lo recomendaría a sus familiares y amigos (Bigné et al., 2007). De acuerdo con el estudio de Bigné, Font y Andreu (2000), si el visitante se va insatisfecho de su viaje, se reduce la probabilidad de repetición y generará una imagen menos positiva del destino entre sus conocidos. Varios autores exponen que la variable clave para obtener la lealtad de un turista es la satisfacción, la cual genera la intención de volver al destino (Yuksel, A., Yuksel, F., y Bilim, 2010; Forgas-Coll, Palau-Saumell, Sánchez-García, y Callarisa-Fiol, 2012). Por ello, la satisfacción es muy importante para recomendar el destino (Hosany y Witham, 2010), debido a que es considerada también como moderadora de la imagen del destino (Chi y Qu, 2008). De igual forma, en varios trabajos se ha reportado el efecto positivo que causa la satisfacción relacionada con la intención de volver a un destino (Zabkar, Brencic, y Dmitrovic, 2009; Chen y Chen, 2011). Das Chagas y Marques Júnior (2011), encontraron que son ocho las dimensiones percibidas de la calidad en los destinos de sol y playa: playas y facilidades, equipamientos públicos, alimentos y bebidas, servicios de transporte, equipamientos del hotel, servicios del hotel, entretenimiento y atractivos, y hospitalidad y accesos. Por lo tanto, es necesario analizar en destinos de sol y playa, como el caso de Montañita, sus establecimientos hoteleros, de restauración y sus equipamientos públicos.

Los viajes de los turistas en destinos de sol y playa son de múltiples propósitos, debido a que los turistas combinan atracciones de la playa con otros lugares de interés situados en el destino (Bujosa, A., Riera, A., y Pons, P. J., 2015), por lo que es conveniente estudiar estos patrones para tener una mayor comprensión de este segmento. En un destino turístico es necesario mejorar sus servicios debido a que si el turista lo visita por sus principales atractivos como la playa y sus actividades recreativas, el turista también tendrá la motivación por visitar otros lugares cercanos y realizar otras actividades.

Por otro lado, en un estudio de Correia, Kozak y Ferradeira (2013) se establece que las congruencias entre las expectativas y el deseo son determinantes en el comportamiento del individuo hacia las vacaciones, lo que hace necesario que los destinos turísticos mejoren sus servicios para superar las expectativas de los turistas y así lograr la satisfacción. También, es preciso estudiar aspectos como la gastronomía, la cultura y el turismo, ya que parecen formar una simbiosis perfecta para turistas, lo que les permite apreciar los diferentes productos y llegar a conocer diferentes destinos, de tal forma que se fomente el desarrollo económico sostenible de determinadas zonas (López-Guzmán y SánchezCañizares, 2012), puesto que el turismo se ve como una posibilidad para el aprovechamiento de recursos, la creación de empleo y un canal para generar desarrollo económico (Zambón y Ramos, 2010). El estudio de las motivaciones y satisfacción de los servicios turísticos podrían brindar información útil para que los actores involucrados en el desarrollo turístico de Montañita puedan fortalecer los atractivos naturales y culturales, y mejorar los servicios turísticos del destino. 


\section{DESCRIPCIÓN DEL ÁREA GEOGRÁFICA}

Del análisis del Producto Interno Bruto (PIB) de Ecuador, se establece la importancia del sector turístico debido a que en las exportaciones no petroleras, este rubro ocupa el tercer lugar, precedido por el sector bananero y camaronero. Por esta razón la investigación está centrada en este sector. Cabe mencionar que de acuerdo al Ministerio de Turismo de Ecuador MINTUR (2016), durante el año 2015 el ingreso de divisas por concepto de viajes y transporte de pasajeros alcanzó la suma de 1.557,4 millones de dólares, observando un incremento del 4,7\% con respecto al año anterior, mostrando un saldo de 532,9 millones en la balanza turística del año 2015. (Tabla 1).

Complementariamente, otro estudio del MINTUR amplía la información acerca de los principales mercados emisores de turistas extranjeros en el año 2015, y estableció que los países de orígenes fueron: Colombia, Estados Unidos y Perú.

\section{Tabla 1}

BALANZA TURÍSTICA ANUAL

\begin{tabular}{|rrrrr|}
\hline AÑO & INGRESOS & \% Var. & EGRESOS & \% Var. \\
\hline 2010 & 786,6 & - & 863,4 & - \\
\hline 2011 & 849,7 & 8 & 916,5 & 6,2 \\
\hline 2012 & $1.038,80$ & 22,3 & 943,6 & 3 \\
2013 & $1.251,20$ & 20,5 & 987,1 & 4,6 \\
\hline 2014 & $1.487,20$ & 18,9 & $1.018,20$ & 3,2 \\
\hline 2015 & $1.557,4$ & 4,7 & $1.024,5$ & 0,4 \\
\hline
\end{tabular}

Fuente: MINTUR 2016 en base a Banco Central del Ecuador.

Asimismo, el MINTUR proporciona datos de los últimos seis años sobre la llegada de turistas extranjeros. En la tabla 2 se evidencia la evolución favorable de turistas internacionales que fueron acogidos. Ecuador en el año 2015 recibió a 1'543.091 de turistas internacionales.

Tabla 2

\section{LLEGADAS DE EXTRANJEROS AL ECUADOR}

\begin{tabular}{|cc|}
\hline AÑ & TOTAL \\
\hline 2010 & $1^{\prime} 047.098$ \\
\hline 2011 & $1^{\prime} 141.137$ \\
\hline 2012 & $1^{\prime} 271.901$ \\
\hline 2013 & $1^{\prime} 366.269$ \\
\hline 2014 & $1^{\prime} 557.006$ \\
\hline 2015 & $1^{\prime} 543.091$ \\
\hline
\end{tabular}

Fuente: elaboración propia en base a MINTUR (2016). 
La investigación se desarrolla en una de las comunas ubicadas dentro de la provincia de Santa Elena de la República del Ecuador. Montañita limita al norte con la Comuna de Olón, al sur con la cabecera parroquial Manglaralto, al este con la Cordillera Chongón Colonche y al oeste con el Océano Pacífico. Está ubicada a 200 km de la ciudad de Guayaquil, convirtiéndose en un destino potencial para aquellos turistas que arriban a esta ciudad, por el aeropuerto internacional José Joaquín de Olmedo. (Figura 1).

\section{Figura 1}

\section{UBICACIÓN GEOGRÁFICA DE MONTAÑITA}
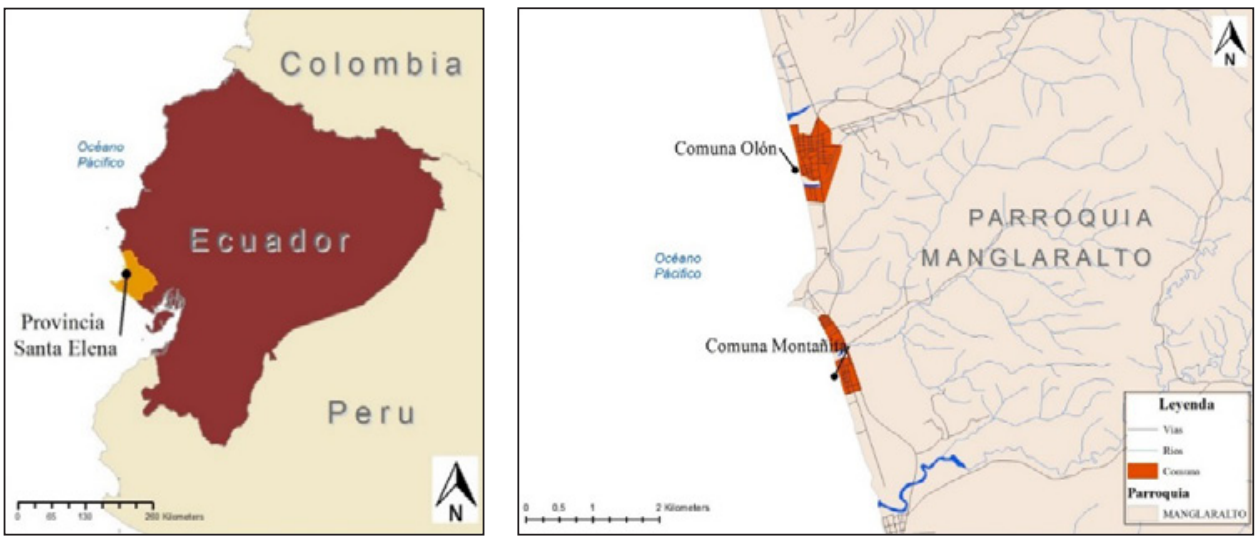

Fuente: Elaboración propia (Utilizando el programa ArcGis 10.0).

La infraestructura que prevalece en Montañita es de estilo moderno e incorpora materiales de la zona como la madera y la hoja de la palma de Tagua (Cade), proporcionando un ambiente agradable para la estadía del turista. La gastronomía del destino se caracteriza por el uso de una gran variedad de mariscos y frutos del mar, combinados con platos internacionales y nacionales. En la década de los sesenta del siglo XX, el lugar se convirtió en un sitio de encuentro para turistas, en su mayoría extranjeros, que venían a disfrutar de la playa, las olas, la diversión, el descanso y el contacto con la naturaleza.

\section{METODOLOGÍA}

La metodología utilizada para la presente investigación empírica se basó en la realización de un trabajo de campo para recolectar la información, para lo cual se utilizó un cuestionario elaborado en base a diferentes trabajos previos sobre el perfil del turista (Correia et al., 2013; López-Guzmán et al., 2015; Guilarte y González, 2016). El trabajo de campo se desarrolló entre los meses de septiembre y octubre del 2015, el cuestionario constaba de 24 preguntas, organizado técnicamente en tres secciones. En la primera sección se requería de información sociodemográfica de los turistas, la segunda sección se enfocaba en las características y motivaciones turísticas del viaje realizado y en la tercera sección el cuestionario 
trataba de la valoración de los servicios turísticos de Montañita. El cuestionario se aplicó en inglés y español, la población objetivo fueron turistas extranjeros mayores de 18 años.

Los turistas fueron encuestados en la playa de Montañita mientras realizaban actividades de recreación o esperaban un servicio de alimentación. Los turistas llenaron las encuestas de manera independiente y los encuestadores estuvieron cerca del encuestado para solventar dudas sobre las preguntas. Se realizó una prueba piloto con 30 encuestas para validar los cuestionarios. Se utilizó una mezcla de técnicas de preguntas para pretender obtener los resultados más aproximados. Así, se utilizaron preguntas cerradas, de opción múltiple y preguntas con una escala de Likert de 5 puntos para valorar la opinión. Se aplicaron 430 cuestionarios, de las cuales 408 fueron válidos, siendo este el tamaño muestral, para lo cual se utilizó la población infinita debido a que no hay un estudio que determine el número de turistas extranjeros que llega a Montañita, con un margen de error de $+/-4.85 \%$, un nivel de confianza del $95 \%$ y una varianza del $50 \%$. (Tabla 3)

\section{Tabla 3 \\ FICHA DE LA INVESTIGACIÓN}

\begin{tabular}{lc}
\hline Población & Turistas extranjeros \\
\hline Área geográfica & Comuna de Montañita \\
\hline Periodo de realización & Septiembre a octubre de 2015 \\
\hline Procedimiento & Muestreo aleatorio simple \\
\hline Nivel de confianza & $95 \%$ \\
\hline Margen de error & $+/-4.85 \%$ \\
Cuestionarios válidos & 408
\end{tabular}

Fuente: elaboración propia.

Los datos recogidos fueron organizados, tabulados y analizados usando el programa SPSS Versión 22. El tratamiento de los datos se realizó a través de la utilización de herramientas estadísticas univariantes y bivariantes.

\section{RESULTADOS Y DISCUSIÓN}

\subsection{Perfil del Turista}

En el presente estudio, la muestra estuvo compuesta de turistas extranjeros. En cuanto al género, el 55,9 \% de turistas fueron hombres y el 44,1 \% fueron mujeres. En cuanto a la procedencia de los turistas extranjeros encuestados, el 35\% eran de América del Sur, el $31 \%$ de Europa y el $24 \%$ de América del Norte. Por tanto, se puede concluir que Montañita como destino turístico recibe turistas procedentes de varios continentes. (Tabla 4). En cuanto al estado civil de los encuestados los mayores grupos los conformaron, el 
$76,8 \%$ de solteros y el 13,2\% de casados. Los dos grupos más representativos de turistas encuestados en cuanto a la edad los conformaron los grupos entre 20 a 29 años con el $62 \%$, y entre 30 a 39 años con el $21,8 \%$. En cuanto al nivel de formación, el 67,9\% de los turistas tenían estudios universitarios y un 19,1\% tenían estudios secundarios, estableciendo la investigación que el grupo mayoritario lo conformaron turistas con estudios universitarios. En cuanto a los ingresos mensuales se puede observar que un 37,4\% tenían ingresos menores a $\$ 700$ y un 17,3\% tenían ingresos entre \$ 700 y \$ 1000 dólares. Sobre la ocupación, el 22,9\% eran estudiantes, seguidos del 19,4 \% que lo conformaron profesionales independientes (Tabla 4).

Tabla 4

CARACTERÍSTICAS SOCIODEMOGRÁFICAS DE LOS TURISTAS EXTRANJEROS

\begin{tabular}{|c|c|c|c|c|c|}
\hline \multicolumn{2}{|c|}{ VARIABLE } & \multirow{3}{*}{$\begin{array}{r}\text { PORCENTAJE } \\
55,9 \% \\
44,1 \%\end{array}$} & \multicolumn{2}{|r|}{ VARIABLE } & \multirow{3}{*}{$\begin{array}{r}\text { PORCENTAJE } \\
3,4 \% \\
62 \%\end{array}$} \\
\hline Sexo & Hombre & & Edad & Menores a 20 años & \\
\hline \multirow{5}{*}{ Educación } & Mujer & & & 20 - 29 años & \\
\hline & Primaria & $1 \%$ & & 30-39 años & $21,8 \%$ \\
\hline & Secundaria & $19,1 \%$ & & 40-49 años & $5,6 \%$ \\
\hline & Universitaria & $67,9 \%$ & & 50-59 años & $3,7 \%$ \\
\hline & Postgrados & $12 \%$ & & 60 y más años. & $3,4 \%$ \\
\hline \multirow{11}{*}{$\begin{array}{l}\text { Lugar de } \\
\text { Procedencia }\end{array}$} & $\begin{array}{l}\text { América del } \\
\text { Sur }\end{array}$ & $35 \%$ & Ingresos & $\begin{array}{l}\text { Menos de } 700 \\
\text { dólares }\end{array}$ & $37,4 \%$ \\
\hline & Europa & $31 \%$ & & 700 a 1.000 dólares & $17,3 \%$ \\
\hline & $\begin{array}{l}\text { América del } \\
\text { Norte }\end{array}$ & $24 \%$ & & 1.001 a 1.500 dólares & $11,8 \%$ \\
\hline & Australia & $5 \%$ & & 1.501 a 2.500 dólares & $12,5 \%$ \\
\hline & Otros & $5 \%$ & & 2.501 a 3.500 dólares & $9 \%$ \\
\hline & & & & Más de 3.500 dólares & $12 \%$ \\
\hline & & & $\begin{array}{l}\text { Estado } \\
\text { Civil }\end{array}$ & Soltero & $76,8 \%$ \\
\hline & & & & Unión libre & $7,8 \%$ \\
\hline & & & & Viudo & $0,5 \%$ \\
\hline & & & & Casado & $13,2 \%$ \\
\hline & & & & Divorciado & $1,7 \%$ \\
\hline
\end{tabular}

Fuente: elaboración propia.

Se ha incluido el coeficiente Chi-Cuadrado de Pearson para determinar la significación de la relación entre dos variables. Se ha detectado asociación entre el nivel de ingreso y 
la edad (coeficiente de Chi-cuadrado de Pearson $=99,041 ; \mathrm{p}=0,000$ ), entre el nivel de ingreso y el nivel de formación (coeficiente de Chi-cuadrado de Pearson $=48,962 ; \mathrm{p}=$ 0,000 ), entre el nivel de ingreso y la ocupación (coeficiente de Chi-cuadrado de Pearson $=104,873 ; \mathrm{p}=0,000)$.

Acerca de la frecuencia de la visita, los turistas declararon que en un porcentaje del $72,20 \%$ era la primera vez que visitaban el destino Montañita, seguidos del 15,7\% que habían visitado el destino de una a dos veces. De acuerdo a los resultados del estudio, el $43,7 \%$ de los turistas viajaron al destino con amigos, mientras que el 27,3\% viajaron en pareja, y el 23,3\% viajaron solos, es decir se puede establecer que es un destino donde la mayoría de turistas llegan entre dos o más personas. Los grupos estaban conformados por el $62,5 \%$ de menos de 3 personas, el 6,4\% grupos entre 4 y 6 personas y el 31,1\% grupos mayores de 6 personas, por lo que es un destino donde llegan en grupos pequeños. En cuanto a la estadía, el grupo de turistas que estuvo entre uno y tres días fue el de mayor porcentaje obtuvo un $36,3 \%$, el segundo grupo lo conformaron las personas que permanecieron de cuatro a seis días con un $27,0 \%$ y aquellos que permanecieron más de nueve días obtuvieron un $27 \%$, lo que indica que hay grupos que se quedan estadías cortas y hay otros grupos que se quedan estadías largas disfrutando del destino. Se ha detectado asociación entre el nivel de ingreso y los días de estadía (coeficiente de Chi-cuadrado de Pearson $=51,991 ; \mathrm{p}=0,000$ ), entre la ocupación y los días de estadía (coeficiente de Chicuadrado de Pearson $=40,836 ; \mathrm{p}=0,017)$, entre el tipo de compañía con la que viaja y los días de estadía (coeficiente de Chi-cuadrado de Pearson $=51,156 ; \mathrm{p}=0,000$ ), entre el número de visitas anteriores y los días de estadía (coeficiente de Chi-cuadrado de Pearson $=27,558 ; \mathrm{p}=0,000)$ entre el número de acompañantes y los días de estadía (coeficiente de Chi-cuadrado de Pearson $=15,583 ; \mathrm{p}=0,016)$.

Acerca del tipo de hospedaje, el $62 \%$ de los turistas se hospedaron en hostales y el 16,20\% en hoteles. Se ha detectado asociación entre el nivel de ingreso y el tipo de hospedaje (coeficiente de Chi-cuadrado de Pearson $=115,341 ; \mathrm{p}=0,000$ ), entre los días de estadía y el tipo de hospedaje (coeficiente de Chi-cuadrado de Pearson $=63,814 ; \mathrm{p}=$ $0,000)$. En cuanto al medio de transporte utilizado para llegar a Montañita, el $80,1 \%$ llegaron en transporte público. El gasto medio del turista en este destino fue de 41,38 dólares por día, lo que evidencia que Montañita es un destino económico para visitarlo. Del total de turistas encuestados, el 65\% recibieron información de amigos sobre el destino Montañita. Así mismo, de todos los encuestados un 19,4\% recibieron información de Internet, y el 7,4\% recibieron información mediante folletos turísticos, lo que indica que Montañita mayormente se promociona a través de boca oreja. La pregunta era de respuesta múltiple y, por tanto, la sumatoria del resultado es superior a $100 \%$.

\subsection{Motivaciones}

En cuanto a las motivaciones que tuvieron los turistas para visitar el destino Montañita, se muestran en la Tabla 5, estudio basada en una Escala de Likert de 5 puntos, siendo 1 - muy poco y 5 - mucho. Las motivaciones más valoradas fueron Disfrutar del sol y la playa con un 4,22, seguidas de descansar con un 3,99 y la menos valorada fue visitar a familiares y amigos con un 1,92. 
Tabla 5

MOTIVACIONES PARA LA VISITA

\begin{tabular}{lcc}
\hline MOTIVACIONES & MEDIA & RANKING \\
\hline Disfrutar del sol y la playa & 4,22 & 1 \\
\hline Descansar & 3,95 & 2 \\
Realizar recorridos en contacto con la naturaleza & 3,43 & 3 \\
Conocer a la comunidad & 3,41 & 4 \\
Disfrutar de la vida nocturna y diversión & 3,40 & 5 \\
\hline Practicar el surf & 3,03 & 6 \\
Otro & 2,47 & 7 \\
Practicar parasailing & 1,93 & 8 \\
Visita a familiares y amigos & 1,92 & 9
\end{tabular}

Fuente: elaboración propia.

Entre los lugares que pensaban visitar los turistas en el destino mientras duraba su estadía, se encuentran el visitar la playa, seguido de acudir a restaurantes donde se pudiera degustar la comida típica de esta zona, la visita a discotecas y bares, la práctica del surf. En este sentido, la playa, se convierte en el mayor atractivo turístico de este lugar, siendo el surf la actividad deportiva con mayor interés que se realiza en la playa. La pregunta era de respuesta múltiple y, por lo tanto la sumatoria del resultado es superior a 100\% (Tabla 6).

\section{Tabla 6}

\section{LUGARES DE INTERES}

\begin{tabular}{lc}
\hline LUGARES & PORCENTAJE \\
\hline Visitar la playa & $91,90 \%$ \\
\hline Restaurantes de comida típica & $55,10 \%$ \\
\hline Discotecas y bares & $53,20 \%$ \\
\hline Lugares para la práctica de surf & $43,40 \%$ \\
Sitios de turismo de naturaleza & $42,30 \%$ \\
Sitios de entretenimiento & $40,90 \%$ \\
\hline Visitar tiendas de artesanías & $38,50 \%$ \\
\hline
\end{tabular}

Fuente: elaboración propia.

En cuanto a la práctica del surf, el estudio determinó que del total de encuestados, el $42 \%$ mencionaron que si practicaban el surf, mientras que el $58 \%$ declararon que no lo practicaban, siendo este resultado un porcentaje considerado para establecer que aunque 
Montañita es reconocida a nivel internacional como destino de surf, de acuerdo con nuestra investigación, además de la motivación para practicar surf, otros viajeros llegan atraídos a este lugar por sus playas. Las principales razones para venir a este destino a practicar este deporte fueron "la temperatura del agua" $(63,6 \%)$ y "el tamaño del oleaje" $(62,4 \%)$, seguidos de la preferencia por la ola de derecha que se produce en esta playa y el beach break, determinando el estudio que Montañita cuenta con características naturales muy importantes para la práctica del surf, por lo que debería de potencializarse este deporte en este destino.

Además, los turistas fueron consultados acerca de si recomendarían a alguien de su ciudad o país visitar Montañita, el 97,5\% de los turistas señalaron que sí lo recomendarían, mientras que el 2,5\% mencionaron que no lo recomendarían, esto indica el alto nivel de satisfacción del turista en el destino. Se analizó las variables sociodemográficas y se encontró que el $60 \%$ del grupo que no recomendarían el destino eran personas mayores de 40 años de edad.

El estudio también consultó a los turistas si regresarían a Montañita en otra ocasión, el 89,8\% de los turistas extranjeros contestaron que si regresarían a este destino, mientras que el 10,2\% contestó que no regresarían, siendo un porcentaje alto el de los turistas que tienen intenciones de regresar por sus principales motivaciones la de disfrutar del sol y la playa y la descansar, estos resultados revelan la lealtad de parte de los turistas hacia este destino. Los turistas que mencionaron que no regresarían están distantes al destino, se encontró que el $63 \%$ provenían del continente Europeo (Tabla 7).

Tabla 7

\section{TURISTAS POR PROCEDENCIA QUE NO REGRESARÍAN}

\begin{tabular}{lc}
\hline CONTINENTE & PORCENTAJE \\
\hline América del Sur & $15 \%$ \\
\hline Europa & $63 \%$ \\
América del Norte & $20 \%$ \\
Australia & $0 \%$ \\
Otros & $2 \%$
\end{tabular}

Fuente: elaboración propia

\subsection{Valoración de los servicios turísticos}

En cuanto a la valoración de los servicios hoteleros, las diferentes variables relacionadas con los servicios hoteleros de Montañita se muestran en la Tabla 8, estudio basada en una Escala de Likert de 5 puntos, siendo 1-muy insatisfecho y 5-muy satisfecho. Así, en los servicios hoteleros las variables más valoradas fueron la ubicación de los hospedajes turísticos $(4,08)$, servicio de acceso a Internet $(3,99)$ y la atención del personal $(3,92)$. Estos aspectos indican que es alta la satisfacción por la ubicación de los establecimien- 
tos hoteleros. Por otra parte, las variables menos valoradas, aunque su satisfacción fue media, fueron el diseño arquitectónico $(3,57)$, la decoración y mobiliario $(3,58)$ y la calidad de la información recibida $(3,69)$; lo que hace necesario que se trabaje en potenciar las variables mayormente valoradas y se mejoren las variables menos valoradas en los establecimientos hoteleros.

\section{Tabla 8}

SATISFACCION DE LOS SERVICIOS HOTELEROS

\begin{tabular}{lcc}
\hline ASPECTOS & MEDIA & RANKING \\
\hline Ubicación del Hotel & 4,08 & 1 \\
\hline Servicio de Internet & 3,99 & 2 \\
Atención del personal & 3,92 & 3 \\
Limpieza & 3,80 & 4 \\
Profesionalismo en el Check-in & 3,74 & 5 \\
Confortabilidad de las camas & 3,72 & 6 \\
Calidad de la información recibida & 3,69 & 7 \\
\hline Decoración y mobiliario & 3,58 & 8 \\
Diseño arquitectónico & 3,57 & 9
\end{tabular}

Fuente: elaboración propia.

Con respecto a la relación entre las principales motivaciones y las diferentes variables de los hoteles, que se muestra en la Tabla 9, analizado a través de una Correlación de Spearman, se encontró que las variables que tienen mayor influencia con la motivación por disfrutar el sol y la playa son el servicio de Internet y la atención del personal, mientras que las variables que tienen mayor influencia con la motivación por descansar son la limpieza, la calidad de la información recibida, el profesionalismo en el Check-in y la atención del personal. En cambio las variables con mayor influencia con la motivación por realizar recorridos en contacto con la naturaleza son la calidad de la información recibida, la limpieza y la decoración y mobiliario (Tabla 8).

En cuanto a los servicios de restauración en la Tabla 10 se muestran los resultados del estudio basada en una Escala de Likert de 5 puntos, siendo 1-muy insatisfecho y 5-muy satisfecho. La calificación de las variables mejor valoradas fueron la calidad de las comidas y bebidas $(3,84)$ y la atención del personal $(3,78)$. Estos resultados indican que es media -alta la satisfacción por la calidad de las comidas y bebidas, siendo la gastronomía un atractivo turístico intangible del destino con mucha potencial para el turismo. Así mismo, los servicios menos valorados, aunque su satisfacción fue media, fueron carta de vinos $(2,59)$ y la presentación de los platos $(3,55)$. Por ello es necesario que se trabaje en potenciar las variables mayormente valoradas y en mejorar las variables menos valoradas en los establecimientos de restauración (Tabla 10). 
Tabla 9

PRINCIPALES MOTIVACIONES CON VARIABLES EN SERVICIOS HOTELEROS (Correlación de Spearman)

\begin{tabular}{|c|c|}
\hline ASPECTOS & CORRELACIÓN \\
\hline \multicolumn{2}{|l|}{ MOTIVACIÓN SOL Y PLAYA } \\
\hline Servicio de internet & $0,140 * *$ \\
\hline Atención del personal & $0,139 * *$ \\
\hline Ubicación del Hotel & $0,128 *$ \\
\hline Diseño arquitectónico & $0,119 *$ \\
\hline \multicolumn{2}{|l|}{ MOTIVACIÓN DESCANSAR } \\
\hline Limpieza & $0,271 * *$ \\
\hline Calidad de la información recibida & $0,230 * *$ \\
\hline Profesionalismo en el Check-in & $0,225 * *$ \\
\hline Atención del personal & $0,217 * *$ \\
\hline Ubicación del Hotel & $0,190 * *$ \\
\hline Confortabilidad de las camas & $0,187 * *$ \\
\hline Servicio de internet & $0,159 * *$ \\
\hline \multicolumn{2}{|c|}{ MOTIVACIÓN RECORRIDOS CON LA NATURALEZA } \\
\hline Calidad de la información recibida & $0,238 * *$ \\
\hline Limpieza & $0,212 * *$ \\
\hline Decoración y mobiliario & $0,190 * *$ \\
\hline Confortabilidad de las camas & $0,117 * *$ \\
\hline
\end{tabular}

Tabla 10

SATISFACCIÓN DE LOS SERVICIOS DE RESTAURANTES

\begin{tabular}{lcc}
\hline ASPECTOS & MEDIA & RANKING \\
\hline Calidad de las comidas y bebidas & 3,84 & 1 \\
\hline Atención del personal & 3,78 & 2 \\
\hline Tiempo de espera & 3,73 & 3 \\
\hline Variedad del menú & 3,71 & 4 \\
\hline Relación calidad/precio & 3,68 & 5 \\
\hline Instalaciones & 3,58 & 6 \\
\hline Presentación de los platos & 3,55 & 7 \\
\hline Carta de vinos & 2,59 & 8 \\
\hline
\end{tabular}

Fuente: elaboración propia. 
Entre los aspectos que los turistas consideran que hay que mejorar en el destino se encuentran el cuidado del lugar, los puntos de información turística, los servicios básicos y la infraestructura. Los aspectos que menos consideraron que hay que mejorar fueron el alquiler de accesorios para la práctica de deportes de sol y playa, seguido de la atención al cliente. Consideremos que es muy importante tener presente estas conclusiones dentro de los planes de desarrollo turísticos del lugar La pregunta era de respuesta múltiple por los tanto la sumatoria del resultado es superior a 100\% (Tabla 11).

Tabla 11

ASPECTOS A MEJORAR EN EL DESTINO

\begin{tabular}{lc}
\hline ASPECTOS & VALORACIÓN \\
\hline Cuidado del Lugar & $45,50 \%$ \\
\hline Puntos de información turística & $38,10 \%$ \\
\hline Servicios básicos & $35,20 \%$ \\
\hline Infraestructura & $29,40 \%$ \\
Gastronomía & $21,50 \%$ \\
\hline Seguridad & $18,30 \%$ \\
Señalización & $17,70 \%$ \\
\hline Espacios de parqueo & $17,50 \%$ \\
Centros de recreación & $16,00 \%$ \\
\hline Atención al cliente & $13,50 \%$ \\
\hline Alquiler de accesorios para práctica de deportes de sol y playa & $12,5 \%$
\end{tabular}

Fuente: elaboración propia.

\section{CONCLUSIONES}

Se concluye al culminar el presente estudio que el analizar el perfil, las motivaciones y el grado de satisfacción del turista trae una amplia información útil para mejorar los servicios turísticos de un destino y, de esta forma, impactar positivamente en la experiencia de los turistas cuando se realiza la visita. Es importante que las diferentes administraciones públicas, tanto nacionales como locales, con responsabilidades en el sector turístico y las empresas privadas trabajen en mejorar el destino y desarrollen estrategias con la finalidad de reforzar las variables más valoradas y, al mismo tiempo, mejoren las variables menos valoradas tanto en los servicios hoteleros como en los servicios de restaurantes.

Los resultados de esta investigación sugieren evidencia importante acerca del perfil del turista que visita Montañita. Así, los turistas al destino llegan de forma variada hombres y mujeres, procedentes de varios continentes, su gran mayoría son solteros, la 
edad comprendida del grupo mayoritario de turistas es entre 20 a 29 años, con estudios universitarios, en cuanto a la ocupación la gran mayoría son estudiantes y profesionales independientes. Montañita es un destino donde los turistas llegan en grupos pequeños, hay grupos que permanecen estadías cortas hasta de tres días y otros que se quedan estadías largas disfrutando del destino. La gran mayoría de turistas se hospedan en hostales y al destino llegan por lo general en transporte público.

El gasto medio del turista por día es de 41,38 dólares, lo que hace que el lugar sea económico para el turista, el destino Montañita generalmente se promociona a través de boca oreja. Las motivaciones más valoradas fueron disfrutar del sol y la playa, seguida de descansar. Entre los lugares que pensaban visitar los turistas se encuentran la playa, siendo esta su mayor atractivo turístico, seguido de visita a restaurantes de comida típica. Las variables más valoradas en cuanto a los servicios de los establecimientos de alojamiento, fue la ubicación de los hospedajes turísticos y la variable más valorada de los restaurantes fue la calidad de las comidas y bebidas, lo que demuestra la importancia de la playa y la riqueza gastronómica del destino.

En base al estudio se evidencia que a Montañita también llegan turistas que no practican el surf lo que muestra que otros viajeros llegan atraídos a este lugar por sus playas. Aquellos turistas que si practicaban el surf mencionaron que lo más importante que los atraía para la práctica del surf era la temperatura del agua y el tamaño del oleaje, lo que evidencia el potencial de sus recursos naturales para la práctica de este deporte.

Los turistas extranjeros que fueron encuestados están dispuestos a recomendar a sus familiares y amigos la visita al destino, lo que evidencia su buena experiencia durante su estadía. Así mismo, la mayoría de los turistas expresaron que si regresarían al destino, lo que indica la buena satisfacción que el turista encuentra en este destino.

Por otro lado, podría ser una buena opción para mejorar la calidad de los servicios turísticos en Montañita y, de esta forma, lograr aumentar la frecuencia de visitas al destino y para que los prestadores de servicios turísticos diseñen productos y ofrezcan servicios turísticos acordes a la demanda y para que las instituciones públicas implementen planes y políticas públicas que impulsen el desarrollo turístico de Montañita. El destino Montañita cuenta con un gran potencial para el turismo de sol y playa y para la práctica del surf lo que hace necesario realizar estudios de este tipo que contribuyan en mejorar la oferta turística del destino.

La principal limitación de este estudio es el periodo temporal en el que se realizó el trabajo de campo. Finalmente, proponemos como futura línea de investigación la percepción de la comunidad local con respecto al desarrollo turístico de este lugar.

\section{BIBLIOGRAFÍA}

ARIZÓN, M.J.B., GARCÉS, S.A. y SANGRÁ, M.M. (2012): "Perfil del turista de festivales: el caso del Festival Internacional de las Culturas Pirineos Sur". Cuadernos de Turismo, no 30, pp. 63-90.

AYALA, H., MARTÍN, R. y MASIQUES, J. (2003). El turismo de sol y playa en el Siglo XXI. Teoría y Práctica del Turismo. Anexos. La Habana: Centro de Estudios Turísticos de la Universidad de La Habana. 
BELTRÁN BUENO, M.Á. (2014). Las motivaciones como factor clave de la conducta turística. Un estudio empírico en el Sureste Español. Murcia: Servicio de Publicaciones de la UCAM.

BLANCO, J.M.C., DÍAZ, A.C., DAUDER, S.G. y SÁEZ, A.M. (2003): “Aproximación psicosocial a la motivación turística: variables implicadas en la elección de Madrid como destino". Estudios Turísticos, n ${ }^{\circ} 158$, pp. 5-42.

BIGNÉ, E., FONT, X. y ANDREU, L. (2000). Marketing de Destinos Turísticos. Análisis y estrategias de desarrollo. Madrid: ESIC.

BIGNÉ, E., SÁNCHEZ, I. y CURRÁS, R. (2007): “El papel de la imagen del destino en la valoración y comportamiento postcompra del turista de sol y playa". Papers de Turisme, ${ }^{\circ}$ 42, pp. 57-73.

BUJOSA, A., RIERA, A. y PONS, P.J. (2015): "Sun-and-beach tourism and the importance of intra-destination movements in mature destinations". Tourism Geographies, vol. 17(5), pp. 780-794.

BRIDA, J.G., AGUIRRE, S.Z. y VELÁSQUEZ, C.M.G. (2010): “Análisis del perfil y satisfacción de los pasajeros de cruceros que visitan el puerto de Cartagena de Indias". Turismo y Desarrollo Local, vol. 3 (8), pp. 1-8.

COHEN, E. (2005): "Principales tendencias en el turismo contemporáneo". Política y Sociedad, vol. 42 (1), pp. 11-24.

CORREIA, A., KOZAK, M. y FERRADEIRA, J. (2013): "From tourist motivations to tourist satisfaction". International Journal of Culture, Tourism and Hospitality Research, vol. 7(4), pp. 411-424.

CHEN, L.J., y CHEN, J.S. (2011): “The motivations and expectations of international volunteer tourists: A case study of Chinese village traditions". Tourism Management, vol. 32, pp. 435-442.

CHI, C.G.Q. y QU, H. (2008): "Examining the structural relationships of destination image, touris satisfaction and destination loyalty: An integrated approach". Tourism Management, vol. 29, pp. 624-636.

DAS CHAGAS, M.M. y MARQUES JÚNIOR, S. (2011): “Dimensiones de la calidad como antecedentes de la satisfacción y fidelidad a los destinos turísticos de sol y playa: Un estudio de caso en Natal, Brasil, sobre la percepción del turista nacional". Estudios y perspectivas en Turismo, vol. 20 (6), pp. 1431-1448.

ESPEJO MARÍN, C. (2011): "Innovación para la competitividad turística en La Manga del Mar Menor (Murcia)". Cuadernos de Turismo, n 27, pp. 321-339

FORGAS-COLL, S., PALAU-SAUMELL, R., SÁNCHEZ-GARCÍA, J. y CALLARISAFIOL, L.J. (2012): "Urban destination loyalty drivers and cross-national moderator effects: The case of Barcelona". Tourism Management, vol. 33, pp. 1309-1320.

GONZÁlEZ, A., SÁNCHEZ, I. y SANZ, S. (2006): Caracterización del turista de sol y playa: una comparativa con el turista rural y cultural. En IX Congreso de Turismo Universidad y Empresa (Sol, playa y turismo residencial), Castellón.

HOSANY, S., y WITHAM, M. (2010): “Dimensions of cruisers' experiences, satisfaction, and intention to recommend". Journal of Travel Research, vol. 49, pp.351-364.

ITB. (2015). ITB World Travel Trends Report 2015/2016. Recuperado de http://www.itbberlin.de/media/itb/itb_dl_all/itb_presse_all/WTTR_ITB2016_8_Web.pdf 
KOZAK, M., BIGNÉ, E. y ANDREU, L. (2003): "Limitations of cross-cultural customer satisfaction researchand recommending alternative methods". Journal of Quality Assurance in Hospitality and Tourism, vol. 4 (3/4), pp. 37-59.

LÓPEZ-GUZMÁN, T., RIBEIRO, M.A., ORGAZ-AGÜERA, F. y MARMOLEJO MARTÍN, J. A. (2015): "El turismo en Cabo Verde". Estudios y Perspectivas en Turismo, vol. 24, pp. 512-528.

LÓPEZ-GUZMÁN, T. y SÁNCHEZ CAÑIZARES, S. (2012): "Culinary tourism in Cordoba (Spain)". British Food Journal, vol. 114 (2), pp. 168-179.

MARTÍNEZ, J.A. (2011): "Factores que influyen en el comportamiento de compra del turista”. TURyDES, Revista de investigación en Turismo y Desarrollo Local, vol. 4 (9), pp. 1-13.

MARTÍNEZ, R. (2001): “Aproximación al estudio del sector turístico almeriense: análisis de la oferta y de la demanda en temporada alta". Cuadernos de Turismo, $\mathrm{n}^{\circ} 7, \mathrm{pp}$. 81-91.

MINISTERIO DE TURISMO DE ECUADOR (2016) Datos estadísticos, 2009-2015. Quito: Servicios Publicados.

MOLINA COLLADO, A., MARTÍN-CONSUEGRA NAVARRO, D., ESTEBAN TALAYA, A. y DÍAZ SÁNCHEZ, E. (2011): “Segmentación de la demanda turística: un análisis aplicado a un destino de turismo cultural". Revista de Análisis Turístico, $\mathrm{n}^{\circ} 4$, pp. 38-48.

OLIVER, R.L. (1980): “A cognitive model of the antecedents and consequences of satisfaction decisions”. Journal of Marketing Research, vol. 14 (4), pp. 460-469.

PÉREZ GUILARTE, Y. y LOIS GONZÁLEZ. R. (2016): "El perfil del visitante en Santiago de Compostela: tradición y actualidad." Cuadernos de Turismo, no 37, pp. 305-322.

RIVAS, J. (2004): Estructura y Economía del Mercado Turístico. Oviedo, Septem Ediciones. URRY, J. (1994): “Time, leisure and social identity". Time \& Society, vol. 3 (2), pp. 131-149. YAGÜE PERALES, R.M. y MARCH CHORDÀ, I. (2004): “Análisis del comportamiento de la demanda de turismo rural desde la experiencia de la comarca El Alto Palancia. Estudios Geográficos, vol. 65, n 254, pp. 121-141.

YUKSEL, A., YUKSEL, F. y BILIM, Y. (2010): “Destination attachment: Effects on customer satisfactionand cognitive, affective and conative loyalty". Tourism Management, vol. 31, pp. 274-284.

ZAMBÓN, G.F. y RAMOS, A. (2010): "El patrimonio cultural como oferta complementaria al turismo de sol y playa. El caso del sudeste Bonaerense. Argentina". Pasos: Revista de turismo y patrimonio cultural, vol. 8 (1), pp. 139-149

ZABKAR, V., BRENCIC, M.M., y DMITROVIC, T. (2009): "Modeling perceived quality, visitor satisfaction and behavioural intentions at the destination level". Tourism Management, vol. 31, pp. 537-546. 
